\title{
A perspective on the obstacles to successful dermatological treatment in Yemen
}

Keywords: yemen, dermatology, obstacles, skin diseases, leishmaniasis

\section{Introduction}

In this report, I will quickly highlight the most common factors that may contribute to improper management plans of skin disorders, particularly chronic dermatoses, in Yemen from my perspective and depending on my own experience managing Yemeni patients with all skin conditions in many locations at both rural and urban health facilities.

Yemen, a country facing major natural and human-made disasters, has a complicated geographical, environmental, topographic and social conditions. It has an estimated population of more 21 million living in only $2 \%$ of its geographical area with a growth rate considered as among the highest in the World; $74 \%$ of them living in rural areas and mountains where the health services are scarce, only $50 \%$ of the total population and $30 \%$ of the rural population has access to public health services, and more than $40 \%$ are below the lower poverty line and suffering froma constantly increasing rate of unemployment. ,

Yemeni rural women usually face additional barriers to seek treatment and, are predominantly the most affected group by skin diseases, and usually face additional barriers to seek treatment.

"Dermatitis, eczematous, and allergic disorders, followed by skin infections and infestations, and pigmentary disorders are the commonest skin disorder groups. Contact dermatitis (allergic and irritant), followed by hyper pigmentations, acne, viral infections, atopic dermatitis, and parasitic infestations topped the list of the most prevalent skin disorders."Leishmaniasis is the most prevalent skin infectious disease in some regions such as Central Yemen. ${ }^{1}$

Climate, occupational, inherited social habits, habitual gender- and age-specific occupational roles, and environmental factors are the main contributors. Serious occupational skin diseases most often prevail among agriculture and construction workers. Most the contagious diseases affect low and lower-middle income populations. ${ }^{1-3}$

\section{Obstacles}

The most common obstacles to a successful dermatological treatment in Yemen the author has concluded, depending on his 15 -year experience treating skin diseases in several rural and urban regions in Yemen, are: the fact that children and women represent the sector suffering thegreatest deficiencies of medical care. Gender inequality in preferential treatment inrural and tribal communities; stigmata associated with some skin disease in females that keep females hidden; drug abuse, self-treatment, and widely-used popular therapies and local healers' methods. The high illiteracy rate, particularly among rural Yemeni females, and the lack of essential knowledge about skin diseases' nature, etiology and primary protective measures; Poverty and malnutrition, which is more aggressive among rural children; the habit of keeping livestock within human habitations intense use of illegal agricultural pesticides particularly in khat shrub
Volume I Issue 3 - 2017

\author{
Mohamed Ahmed Al Kamel \\ Department of Dermatology, Sana'a University Hospital,Yemen
}

Correspondence: Mohamed Ahmed Al Kamel, Regional Leishmaniasis Control Center, P. O. Box 12692, Sana'a, Yemen, Emaildralkamel@hotmail.com

Received: August 17, 2017| Published:September 19, 2017

cultivation (the gross domestic product of the agricultural sector); prolonged sun exposure among rural inhabitants; poor irrigation systems and inadequate water supply; weak health infrastructures; lack of reservoir/vector control programs; inadequate housing, poor sanitation, and bad hygiene; increased transportation and medication cost; physical barriers, such as poor geographical access to health facilities; conflicts and displacement of population.

\section{Conclusion}

Human is a "child of the earth" and every individual's skin is a mirror that reflects his or her overall health condition, as well the surrounding environment. The above-mentioned obstacles to successful medical treatment of dermatological conditions in Yemen may accentuate the burden of skin diseases and often result in treatment failure, hidden diseases and high recurrence rate that may depress, debilitate, deform or even kill. Understanding these barriers is very important before organizing any skin disease-control program in underserved region. ${ }^{4}$

\section{Acknowledgements}

None.

\section{Conflict of interest}

The author declares no conflict of interest.

\section{References}

1. Al Kamel MA. Spectrum of winter dermatoses in rural Yemen. International Journal of Dermatology. 2016;55(5):512-517.

2. Abdul Ghani R. Combat against neglected parasitic diseases in Yemen: the need for mapping as a prerequisite for elimination. Yemeni J Med Sci. 2016;10:1-5.

3. Al Kamel MA. Impact of leishmaniasis in women: a practical review with an update on my ISD-supported initiative to combat leishmaniasis in Yemen (ELYP). International Journal of Women's Dermatology. 2016;2(3):93-101.

4. Khatri ML. Spectrum of skin diseases in Yemen (Hajjah and adjacent region). Int J Dermatol. 2004;43(8):580-585. 